\title{
EXISTENCE AND ASYMPTOTIC PERIODICITY OF SOLUTIONS FOR NEUTRAL INTEGRO-DIFFERENTIAL EVOLUTION EQUATIONS WITH INFINITE DELAY
}

\author{
JiANBO ZHU* - XIANLONG FU**, \\ (Communicated by Michal Fečkan)
}

\begin{abstract}
In this work, making use of the theory of resolvent operators and Banach fixed point theorem, we first discuss the existence and regularity of mild solutions for neutral partial functional integro-differential equations with infinite delay. We assume that the linear part of the considered equation generates a resolvent operator and the nonlinear function satisfies Lipschitz conditions. Then we investigate the asymptotic periodicity of mild solutions under asymptotic periodic assumption on the nonlinear function. The obtained results extend somewhat the related conclusions in literature. In the end, an example is presented to illustrate the obtained results.
\end{abstract}

$$
\text { (C) } 2022
$$

Mathematical Institute Slovak Academy of Sciences

\section{Introduction}

In this paper, we study the existence, regularity and asymptotic periodicity of solutions for neutral functional integro-differential evolution equations with infinite delay of the form

$$
\left\{\begin{array}{l}
\frac{\mathrm{d}}{\mathrm{d} t}\left[x(t)+\int_{0}^{t} N(t-s) x(s) \mathrm{d} s\right]=A\left[x(t)+\int_{0}^{t} F(t-s) x(s) \mathrm{d} s\right]+f\left(t, x_{t}\right), \quad t \geq 0, \\
x_{0}=\phi \in \mathscr{B},
\end{array}\right.
$$

where $x(\cdot)$ is the state variable taking values in a Banach space $X$ and the notation $x_{t}$ represents the functional defined by $x_{t}:(-\infty, 0] \rightarrow X, x_{t}(\theta)=x(t+\theta)$, belonging to some abstract phase space $\mathscr{B}$ which is defined axiomatically. The operator $(A, D(A))$ is the infinitesimal generator of an analytic semigroup on $X$, while $F(t): D(A) \rightarrow D(A)$ and $N(t): X \rightarrow X$ are two families of bounded linear operators. The nonlinear function $f(\cdot, \cdot)$ is to be specified later.

Neutral partial integro-differential equations without or with time delay arise in various areas. For example, the following heat conduction system, see $3,6,7,30$, has been frequently used to

2020 Mathematics Subject Classification: Primary 34K30, 34K40, 35B10; Secondary 45K05; 47N20.

Keywords: Neutral integro-differential evolution equation, asymptotic periodicity, infinite delay, resolvent operator.

This work was supported by NSF of China, Science and Technology Commission of Shanghai Municipality (STCSM) (grant No. 18dz2271000).

${ }^{\mathrm{c}}$ Corresponding author. 
describe the evolution of the temperature in different types of materials with fading memory,

$$
\left\{\begin{array}{cc}
\frac{\partial}{\partial t}\left[u(t, x)+\int_{-\infty}^{t} k_{1}(t-s) u(s, x) \mathrm{d} s\right]= & c \Delta u(t, x)+\int_{-\infty}^{t} k_{2}(t-s) \triangle u(s, x) \mathrm{d} s \\
& +f(t, u(t, x)), \quad t \geq 0, \\
u(t, x)=0, \quad x \in \partial \Omega . &
\end{array}\right.
$$

In this system, $\Omega \subset \mathbb{R}^{n}$ is open, bounded and has smooth boundary, $(t, x) \in[0, \infty) \times \Omega, u(t, x)$ represents the temperature in $x$ at time $t, c$ is a physical constant and $k_{i}: \mathbb{R} \rightarrow \mathbb{R}, i=1,2$, are functions of the internal energy and the heat flux relaxation respectively, and the internal energy here the heat flux terms are described as functionals of $u$ and $\Delta u$. For more models of partial integro-differential equations and related applications we refer to the paper 22 and the monographs 22 38. One of the the important way to deal with partial integro-differential equations is to transform them into (neutral) abstract integro-differential evolution equations in Banach space, and then study the abstract systems by applying theory of resolvent operators. Actually, by assuming the solution $u$ is known on $(-\infty, 0]$. we can transform 1.2 into the abstract neutral integro-differential evolution equation of the following form

$$
\frac{\mathrm{d}}{\mathrm{d} t}\left[u(t)+F\left(t, u_{t}\right)\right]=A u(t)+\int_{0}^{t} B(t-s) u(s) \mathrm{d} s+f\left(t, u_{t}\right) \quad \text { for } t \geq 0 .
$$

In [10], it was illustrated that the equation

$$
\frac{\mathrm{d} u(t)}{\mathrm{d} t}=A\left[u(t)+\int_{-\infty}^{t} K(t-s) u(s) \mathrm{d} s\right]+\int_{-\infty}^{t} F(t-s) u(s) \mathrm{d} s \quad \text { for } t \geq 0
$$

can be regarded as the abstract formulation of the model proposed and studied by Coleman and Gurtin 8, Gurtin and Pipkin 20], and Miller 31] for the heat flow in a rigid isotropic viscoelastic material in the elastic case, and the model in thermoviscoelasticity considered by Leugering [28.

An important approach to study the above abstract equations is the theory of resolvent operators founded in 17 - 19. Grimmer et al. proved in [17 - 19 that there is a (unique) resolvent operator $(R(t))_{t \geq 0}$ associated to the linear homogeneous equation

$$
\left\{\begin{array}{l}
v^{\prime}(t)=A v(t)+\int_{0}^{t} \gamma(t-s) v(s) \mathrm{d} s \quad \text { for } t \geq 0, \\
v(0)=v_{0} \in X
\end{array}\right.
$$

Based on it the authors obtained the representation, existence, and uniqueness of mild solutions of the following inhomogeneous integro-differential evolution equation in Banach space $X$

$$
\left\{\begin{array}{l}
v^{\prime}(t)=A v(t)+\int_{0}^{t} \gamma(t-s) v(s) \mathrm{d} s+f(t) \quad \text { for } t \geq 0, \\
v(0)=v_{0} \in X
\end{array}\right.
$$

via the resolvent operator, where $f: \mathbb{R}^{+} \rightarrow X$ is a continuous function. Actually, the resolvent operator, replacing the role of $C_{0}$-semigroup for evolution equations, plays an essential role in solving Eq. 1.3 in weak and strict senses. Based on these important works, in these years much works on various topics, such as existence, regularity and stability of solutions and control problems, for semilinear integro-differential evolution equations has been done by many authors 


\section{EXISTENCE AND ASYMPTOTIC PERIODICITY OF SOLUTIONS}

through applying the theory of resolvent operator, see $4,5,11,14,16,32,40$ and the references therein.

On the other hand, existence of periodic solutions is one of the most interesting and important topics in the qualitative theory of differential equations due to their significance in practical sciences. Many contributions on the existence of periodic solutions for differential equations have been made. Meanwhile, the concept of asymptotically $\omega$-periodic solutions is more general than periodic solutions and, from view of applications, asymptotically periodic systems describe the models more realistically and accurately than periodic ones. The notion of $S$-asymptotically $\omega$-periodic functions was introduced by Henríquez et al. in 25,26] (i.e. a bounded continuous function $f(\cdot)$ is called to be $S$-asymptotically $\omega$-periodic if there exists $\omega>0$ such that $\left.\lim _{t \rightarrow \infty}\|f(t+\omega)-f(t)\|=0\right)$ and it is related to and more general than that of asymptotic $\omega$-periodic functions. Moreover, the authors have established there a relationship between $S$-asymptotically $\omega$-periodic functions and the class of asymptotically $\omega$-periodic functions. In these years this topic is attracting much attention increasing interest of many researchers, some recent works of this area can be found in [1, 9, 12, 24, 29, 35, 37, 39 among others. Particularly, Dos Santos and Henríquez 37] investigated existence of $S$-asymptotically $\omega$-periodic solutions for neutral integro-differential equations of the following form

$$
\left\{\begin{array}{l}
\frac{\mathrm{d}}{\mathrm{d} t}\left[x(t)+\int_{0}^{t} N(t-s) x(s) \mathrm{d} s\right]=A x(t)+\int_{0}^{t} B(t-s) x(s) \mathrm{d} s+f(t, x(t)) \quad \text { for } t \geq 0, \\
x(0)=x_{0},
\end{array}\right.
$$

via the theory of resolvent operators associated to the linear homogeneous equation

$$
\left\{\begin{array}{l}
\frac{\mathrm{d}}{\mathrm{d} t}\left[x(t)+\int_{0}^{t} N(t-s) x(s) \mathrm{d} s\right]=A x(t)+\int_{0}^{t} B(t-s) x(s) \mathrm{d} s \quad \text { for } t \geq 0, \\
x(0)=x_{0},
\end{array}\right.
$$

which was founded in [36]. In [36] the authors first established the theory of resolvent operators for (1.4) and then they discussed the existence and uniqueness of solutions for a semilinear neutral integro-differential equation with infinite delay.

To the best of our knowledge, the regularity and asymptotic periodicity of mild solutions for the neutral partial functional integro-differential equation (1.1) are untreated topics in the literature up to know. The purpose of this paper is to explore the existence, regularity and $S$-asymptotic periodicity of solutions for the equation (1.1). The main tool in our discussion is also the theory of resolvent operators for neutral linear integro-differential systems of (1.1) established in [36. We will represent the global mild solutions of Eq. (1.1) by the resolvent operator $R(t)$ and analyze existence and uniqueness of solutions by using theory of resolvent operator and Banach fixed point theorem. Following this we further study the regularity of mild solutions for Eq. (1.1) under some smoothness conditions. It is seen that the results obtained in this part are better than those in 14, 16, 27 due to the application of the newly theory of resolvent operators. More precisely, other than in 14, 16, 27, we do not impose here any smoothness condition on the neutral term. At last, we study the existence and uniqueness of $S$-asymptotically $\omega$-periodic solutions assuming the nonlinear function is so. The results are proved by utilizing Schauder's fixed point theorem and Banach fixed point theorem, respectively, for the different cases that the resolvent operator is compact and non-compact. We point out here that, compared to 29, 37, the conditions for the existence of $S$-asymptotically $\omega$-periodic solutions are somewhat weaker (see Theorem 4.1 and 
Theorem 4.2). Clearly, our obtained results in this article extend and develop the existing results in literature mentioned above.

Subsequently the paper is organized as follows. In Section 2, we state briefly the basic theory of analytic semigroup and resolvent operators. In Section 3 we discuss the existence and regularity of global mild solutions of (1.1). The existence and uniqueness of $S$-asymptotically $\omega$-periodic solutions is then studied in Section 4. Finally, in Section 5, we provide an example to illustrate the applications of the obtained results.

\section{Preliminaries}

In this section, we present some preliminary facts on theory of analytic semigroup and resolvent operators to be used in this paper.

Let $(X,\|\cdot\|$ and $(Z,\|\cdot\|)$ be two Banach spaces, we denote by $\mathscr{L}(X, Z)$ the Banach space of bounded linear operators from $X$ into $Z$ endowed with the general operator norm and we abbreviate this notation to $\mathscr{L}(X)$ when $X=Z$. For a linear operator $A: D(A) \subseteq X \rightarrow X$, let $Y$ be the Banach space $\left(D(A),\|\cdot\|_{1}\right)$ with the graph norm $\|x\|_{1}=\|A x\|+\|x\|$, for $x \in D(A)$. Hereafter $C([0, T], X)$ denotes the Banach space consisting of continuous functions from $[0, T]$ to $X$ with the norm

$$
\|x\|_{C}=\sup _{0 \leq t \leq T}\|x(t)\|, \quad x \in C([0, T], X) .
$$

$C_{b}([0, \infty) ; X)$ denotes the Banach space consisting of bounded and continuous functions from $[0, \infty)$ to $X$ with $\|\cdot\|_{\infty}$ defined by $\|x\|_{\infty}=\sup _{t>0}\|x(t)\|$.

Next we present briefly the basic theory of resolvent operators for the following neutral integrodifferential equation associated to Eq. (1.1).

$$
\left\{\begin{array}{l}
\frac{\mathrm{d}}{\mathrm{d} t}\left[x(t)+\int_{0}^{t} N(t-s) x(s) \mathrm{d} s\right]=A\left[x(t)+\int_{0}^{t} F(t-s) x(s) \mathrm{d} s\right], \\
x(0)=x_{0} \in X
\end{array}\right.
$$

Definition 1. A one-parameter family of bounded linear operators $(R(t))_{t \geq 0}$ on $X$ is called a resolvent operator for Eq. 2.1) if it verifies the following conditions.

(i) The function $R(\cdot):[0, \infty) \rightarrow \mathscr{L}(X)$ is strongly continuous, and there exist $M_{0} \geq 1$ and $\omega \in \mathbb{R}$, such that $\|R(t)\| \leq M_{0} \mathrm{e}^{\omega t}$ for $t \geq 0$;

(ii) $R(0)=I$;

(iii) For $x \in Y, R(\cdot) x \in C^{1}((0, \infty), X) \cap C([0, \infty), Y)$, and

$$
\begin{aligned}
& \frac{\mathrm{d}}{\mathrm{d} t}\left[R(t) x+\int_{0}^{t} N(t-s) R(s) x \mathrm{~d} s\right]=A\left[R(t) x+\int_{0}^{t} F(t-s) R(s) x \mathrm{~d} s\right], \\
& \frac{\mathrm{d}}{\mathrm{d} t}\left[R(t) x+\int_{0}^{t} R(t-s) N(s) x \mathrm{~d} s\right]=R(t) A x+\int_{0}^{t} R(t-s) A F(s) x \mathrm{~d} s,
\end{aligned}
$$

for each $t \geq 0$.

We assume for the operators in Eq. 1.1) (or (2.1) ) that the following conditions are fulfilled. 


\section{EXISTENCE AND ASYMPTOTIC PERIODICITY OF SOLUTIONS}

$\left(V_{1}\right)$ There are constants $M_{0}>0$ and $\theta \in\left(\frac{\pi}{2}, \pi\right)$ such that

$$
\rho(A) \supseteq \Lambda_{\theta}=\{\lambda \in \mathbb{C}:|\arg (\lambda)|<\theta\} \text { and }\|R(\lambda, A)\| \leq M_{0}|\lambda|^{-1}, \lambda \in \Lambda_{\theta} .
$$

Hence in this case the operator $A$ generates an analytic semigroup $(T(t))_{t \geq 0}$ on $X$ (see 13, 34]).

$\left(V_{2}\right)$ The function $N:[0, \infty) \rightarrow \mathscr{L}(X)$ is strongly continuous and $\hat{N}(\lambda) x$ is absolutely convergent for $x \in X$ and $\operatorname{Re}(\lambda)>0$. There exist $\beta>0$ and an analytical extension of $\hat{N}(\lambda)$ (still denoted by $\hat{N}(\lambda))$ to $\Lambda_{\theta}$ such that $\|\hat{N}(\lambda) x\| \leq N_{0}|\lambda|^{-\beta}\|x\|_{1}$ for each $\lambda \in \Lambda_{\theta}$ and $x \in D(A)$. Here $N_{0}$ is a constant, the notation $\hat{f}(\lambda)$ represents the Laplace transform of $f(t)$.

$\left(V_{3}\right)$ For all $t \geq 0, F(t) \in \mathscr{L}(X) . F(t): X \rightarrow D(A)$, and $A F(\cdot) x$ is strongly measurable on $(0, \infty)$ for any $x \in X$. There exists a $b(\cdot) \in L_{\text {loc }}^{1}\left(R^{+}\right)$such that $\hat{b}(\lambda)$ exists for $\operatorname{Re} \lambda>0$ and $\|A F(t) x\| \leq b(t)\|x\|_{1}$ for all $t>0$ and $x \in D(A)$. Moreover, the operator valued function $\hat{F}: \Lambda_{\frac{\pi}{2}} \rightarrow \mathscr{L}(Y)$ has an analytical extension (still denoted by $\hat{F}$ ) to $\Lambda_{\theta}$ such that $\|A \hat{F}(\lambda) x\| \leq\|A \hat{F}(\lambda)\|\|x\|_{1}$ for all $x \in D(A)$, and $\|A \hat{F}(\lambda)\| \rightarrow 0$ as $|\lambda| \rightarrow \infty$.

$\left(V_{4}\right)$ There exist a subspace $D \subseteq D(A)$ dense in $Y$ and positive constants $C_{i}, i=1,2$, such that $A(D) \subseteq D(A), \hat{F}(\lambda)(D) \subseteq D, \hat{N}(\lambda)(D) \subseteq D(A)$, and $\|A \hat{F}(\lambda) x\|_{1} \leq C_{1}\|x\|_{1},\|\hat{N}(\lambda) x\|_{1} \leq$ $C_{2}|\lambda|^{-\beta}\|x\|_{1}$ for every $x \in D$ and $\lambda \in \Lambda_{\theta}$.

Observe that the above assumptions guarantee that $A F(\cdot)$ verifies the conditions of the family $B(\cdot)$ in [36]. Hence, according to [36], we infer readily that, under the hypotheses $\left(V_{1}\right)-\left(V_{4}\right)$ there is a resolvent operator $R(t)$ for the linear part of (1.1) (i.e. (2.1)), which is given by

$$
R(t)=\left\{\begin{array}{l}
\frac{1}{2 \pi \mathrm{i}} \int_{\Gamma_{r, \vartheta}} \mathrm{e}^{\lambda t} G(\lambda) \mathrm{d} \lambda, \quad t>0, \\
I, \quad t=0,
\end{array}\right.
$$

where $G(\lambda)=(\lambda I+\lambda \hat{N}(\lambda)-A-A \hat{F}(\lambda))^{-1}$ satisfying $\|G(\lambda)\| \leq M_{0}\left|\lambda^{-1}\right|$ for $\lambda \in \Lambda_{r, \vartheta}$ and some $M_{0}>0$. We put $\Omega(G)=\left\{\lambda \in \sum_{F} \cap \sum_{N}: \lambda I+\lambda \hat{N}(\lambda)-A-A \hat{F}(\lambda)\right.$ is invertible and $\left.G(\lambda) \in \mathscr{L}(X)\right\}$, $\sigma(G)=\mathbb{C} \backslash \Omega(G)$ and $W_{G}=\sup \{\operatorname{Re}(\lambda): \lambda \in \sigma(G)\}$. Here the notations $\sum_{F}$ and $\sum_{N}$ stand for the domain of maximal extension of the operators $\hat{F}(\cdot)$ and $\hat{N}(\cdot)$ on $\mathbb{C}$, respectively. The region $\Lambda_{r, \vartheta}=\{\lambda \in \mathbb{C} \backslash\{0\}:|\lambda|>r,|\arg (\lambda)|<\vartheta\}$, and $\Gamma_{r, \vartheta}=\bigcup_{i=1}^{3} \Gamma_{r, \vartheta}^{i}$, the curves $\Gamma_{r, \vartheta}^{i}, i=1,2,3$, are given respectively by

$$
\begin{aligned}
& \Gamma_{r, \vartheta}^{1}=\left\{t \mathrm{e}^{\mathrm{i} \vartheta}: t \geq r\right\}, \\
& \Gamma_{r, \vartheta}^{2}=\left\{r \mathrm{e}^{\mathrm{i} \xi}:-\vartheta \leq \xi \leq \vartheta\right\}, \\
& \Gamma_{r, \vartheta}^{3}=\left\{t \mathrm{e}^{-\mathrm{i} \vartheta}: t \geq r\right\},
\end{aligned}
$$

for $r>0, \vartheta \in\left(\frac{\pi}{2}, \theta\right)$ being fixed numbers. These curves are oriented so that $\operatorname{Im}(\lambda)$ is increasing.

The following theorem founded in [37] will be used in this paper.

THEOREM 2.1. If $W_{G}<0$, then there exist constants $M \geq 1$ and $\gamma>0$ such that

$$
\|R(t)\| \leq M \mathrm{e}^{-\gamma t}, \quad t \geq 0 .
$$

In this paper, we will use an axiomatic definition of the phase space $\mathscr{B}$ introduced by Hale and Kato in 21] and follow the terminology used in [23. Thus, $\mathscr{B}$ is a linear space of functions mapping $(-\infty, 0]$ into $X$ endowed with a seminorm $\|\cdot\|_{\mathscr{B}}$. We assume that $\mathscr{B}$ satisfies the following axioms: 
(A) If $x:(-\infty, \sigma+a) \rightarrow X, a>0$ is continuous on $[\sigma, \sigma+a)$ and $x_{\sigma} \in \mathscr{B}$, then for every $t \in[\sigma, \sigma+a)$ the following conditions hold:

(i) $x_{t}$ is in $\mathscr{B}$.

(ii) $\|x(t)\| \leq H\left\|x_{t}\right\|_{\mathscr{B}}$.

(iii) $\left\|x_{t}\right\|_{\mathscr{B}} \leq K(t-\sigma) \sup \{\|x(s)\|: \sigma \leq s \leq t\}+M(t-\sigma)\left\|x_{\sigma}\right\|_{\mathscr{B}}$.

Here $H \geq 0$ is a constant, $K, M:[0,+\infty) \rightarrow[0,+\infty), K(\cdot)$ is continuous and $M(\cdot)$ is locally bounded, and $H, K(\cdot), M(\cdot)$ are independent of $x(\cdot)$.

$\left(A_{1}\right)$ For the function $x(\cdot)$ in $(A)$, the function $t \rightarrow x_{t}$ is continuous from $[\sigma, \sigma+a)$ into $\mathscr{B}$.

$(B)$ The space $\mathscr{B}$ is complete.

For our later discussion we also need some additional properties of the space $\mathscr{B}$. Let $C_{00}$ be the space of continuous functions from $(-\infty, 0]$ into $X$ with compact support. Then from 23 : Proposition 2.1], $C_{00} \subseteq \mathscr{B}$. We assume that the phase space $\mathscr{B}$ satisfies additionally the following axioms:

$\left(C_{1}\right)$ If $\left\{\phi^{n}\right\}$ is a Cauchy sequence in $\mathscr{B}$ and converges compactly to $\phi$ on $(-\infty, 0]$, then $\phi \in \mathscr{B}$ and $\lim _{n \rightarrow+\infty}\left\|\phi^{n}-\phi\right\|_{\mathscr{B}}=0$.

$\left(C_{2}\right)$ If a uniformly bounded sequence $\left\{\phi^{n}(\theta)\right\}$ in $C_{00}$ converges to a function $\phi(\theta)$ compactly on $(-\infty, 0]$, then $\phi \in \mathscr{B}$ and $\lim _{n \rightarrow+\infty}\left\|\phi^{n}-\phi\right\|_{\mathscr{B}}=0$.

Thus we have that

Lemma 2.1. If the phase space $\mathscr{B}$ satisfies axiom $\left(C_{2}\right)$, then $C_{b}((-\infty, 0], X)$ is continuously imbedded into $\mathscr{B}$, and there exists a constant $J>0$ such that $\|\phi\|_{\mathscr{B}} \leq J\|\phi\|_{\infty^{\prime}}$ for all $\phi \in C_{b}((-\infty, 0], X)$.

In addition, from [33], one has that

Lemma 2.2. Let $\mathscr{B}$ satisfy axiom $\left(C_{1}\right)$ and $f:[0, a] \rightarrow \mathscr{B}$, be a continuous function such that $f(t)(\theta)$ is continuous for $(t, \theta) \in[0, a] \times(-\infty, 0]$. Then

$$
\left(\int_{0}^{a} f(t) \mathrm{d} t\right)(\theta)=\int_{0}^{a} f(t)(\theta) \mathrm{d} t \quad \text { for } \theta \in(-\infty, 0] .
$$

Finally we introduce the concept of fading memory spaces. To do this, let $\mathscr{B}_{0}=\{\phi \in \mathscr{B}$ : $\phi(0)=0\}$ which is a closed subspace of the space $\mathscr{B}$, and let the operator $S_{0}(t)$ on $\mathscr{B}_{0}$ be defined by

$$
\left[S_{0}(t) \phi\right](\theta)=\left\{\begin{array}{lc}
0 & -t \leq \theta \leq 0, \\
\phi(t+\theta) & \theta<-t,
\end{array}\right.
$$

for $\phi \in \mathscr{B}_{0}$. Clearly, the operator family $S_{0}(t), t \geq 0$, is a $C_{0}$-semigroup on $\mathscr{B}_{0}$.

Definition 2. The space $\mathscr{B}$ is called a fading memory space if it additionally verifies Axioms $\left(C_{2}\right)$, and $S_{0}(t) \phi \rightarrow 0$ as $t \rightarrow \infty$ for each $\phi \in \mathscr{B}_{0}$.

Remark 1. According to [23], if $\mathscr{B}$ is a fading memory space, then there exist $K^{*}, M^{*}>0$, such that $K(\cdot)$ and $M(\cdot)$ in $(A)$-(iii) verify $K^{*}=\sup _{t \geq 0}\|K(t)\|$ and $M^{*}=\sup _{t \geq 0}\|M(t)\|$. In this condition, we can choose $J=K^{*}$, here $J$ is the constant in Lemma 2.1 .

\section{Existence and regularity of solutions}

The purpose of this section is to study the existence, uniqueness and regularity of mild solutions for the equation 1.1) on $(-\infty, \infty)$. 


\section{EXISTENCE AND ASYMPTOTIC PERIODICITY OF SOLUTIONS}

\subsection{Existence of solutions}

First we discuss in this subsection the existence and uniqueness of mild solutions for Eq. 1.1). The mild solutions of Eq. 1.1) are defined as:

Definition 3. A function $x: \mathbb{R} \rightarrow X$ is said to be mild solution of Eq. (1.1), if $x_{0}=\phi, x(\cdot)$ is continuous on $[0, \infty)$ and satisfies the integral equation

$$
x(t)=R(t) \phi(0)+\int_{0}^{t} R(t-s) f\left(s, x_{s}\right) \mathrm{d} s \quad \text { for } t \geq 0 .
$$

To guarantee the existence of mild solutions, we impose the following restrictions on the function $f(\cdot, \cdot)$ in Eq. (1.1).

$\left(H_{1}\right)$ The function $f:[0, \infty) \times \mathscr{B} \rightarrow X$ is continuous and there is a constant $L_{1}>0$ such that

$$
\left\|f\left(t, \phi_{1}\right)-f\left(t, \phi_{2}\right)\right\| \leq L_{1}\left\|\phi_{1}-\phi_{2}\right\|_{\mathscr{B}}
$$

for any $t \in[0, \infty), \phi_{1}, \phi_{2} \in \mathscr{B}$, and the inequality

$$
\|f(t, \phi)\| \leq L_{1}\|\phi\|_{\mathscr{B}}, \quad t \in[0, \infty), \phi \in \mathscr{B}
$$

holds.

THEOREM 3.1. Suppose that $\mathscr{B}$ is a fading memory space, and the assumptions $\left(H_{1}\right)$ and $(2.4)$ hold true. Then, for any $\phi \in \mathscr{B}$, the Cauchy problem (1.1) has a unique mild solution on $(-\infty, \infty)$.

Proof. Let $T>0$ be small enough such that $K^{*} L_{1} M T<1$. We define $\mathbb{D}_{\phi}=\{x \in C([0, T], X)$ : $x(0)=\phi(0)\}$ and consider the operator $\Gamma: \mathbb{D}_{\phi} \rightarrow \mathbb{D}_{\phi}$ given by

$$
(\Gamma x)(t)=R(t) \phi(0)+\int_{0}^{t} R(t-s) f\left(s, x_{s}\right) \mathrm{d} s .
$$

Here we identify $x$ with its extension to $(-\infty, 0]$, by $x(\theta)=\phi(\theta)$ for $\theta \leq 0$. Clearly, $\Gamma\left(\mathbb{D}_{\phi}\right) \subset \mathbb{D}_{\phi}$.

We can show easily that $\Gamma$ is a contraction on $\mathbb{D}_{\phi}$. In fact, let $x, y \in \mathbb{D}_{\phi}$, we find

$$
\begin{aligned}
\|(\Gamma x)(t)-(\Gamma y)(t)\| & \leq L_{1} M \int_{0}^{t}\left\|x_{s}-y_{s}\right\|_{\mathscr{B}} \mathrm{d} s \\
& \leq K^{*} L_{1} M T\|x-y\|_{C},
\end{aligned}
$$

for all $0 \leq t \leq T$, that is

$$
\|\Gamma x-\Gamma y\|_{C} \leq K^{*} L_{1} M T\|x-y\|_{C} .
$$

Therefore, we conclude that there exists a fixed point $x(\cdot)$ for $\Gamma$ on $C([0, T], X)$ by Banach fixed point theorem. Now we put

$$
x^{1}(t)= \begin{cases}x(t) & t \in[0, T] \\ \phi(t) & t \in(-\infty, 0],\end{cases}
$$

then $x^{1}(t)$ is clearly a mild solution of problem 1.1$)$ on $(-\infty, T]$. 
Next we extend the solutions of Eq. 1.1 to $(-\infty, 2 T]$. To do so, we define $\phi^{1}=x_{T}^{1}$ and consider the equation

$$
\left\{\begin{array}{l}
\frac{\mathrm{d}}{\mathrm{d} t}\left[y(t)+\int_{0}^{t} N(t-s) y(s) \mathrm{d} s\right]=A\left[y(t)+\int_{0}^{t} F(t-s) y(s) \mathrm{d} s\right]+f\left(t, y_{t}\right), \quad t \in[T, 2 T], \\
y_{T}=\phi^{1} \in \mathscr{B},
\end{array}\right.
$$

The mild solutions of System 3.2 are given by

$$
\left\{\begin{array}{l}
y(t)=R(t-T) \phi^{1}(0)+\int_{T}^{t} R(t-s) f\left(s, y_{s}\right) \mathrm{d} s, \quad t \in[T, 2 T] \\
y_{T}=\phi^{1}
\end{array}\right.
$$

Here we also identify $y$ with its extension to $(-\infty, T]$, which is defined by $y(T+\theta)=\phi^{1}(\theta)$ for $\theta \leq 0$.

Put $\mathbb{D}_{\phi}^{\prime \prime}=\left\{y \in C([T, 2 T], X): y(T)=\phi^{1}(0)\right\}$ and define the operator $\Gamma_{1}$ on $\mathbb{D}_{\phi}^{\prime \prime}$ by

$$
\left(\Gamma_{1} y\right)(t)=R(t-T) \phi^{1}(0)+\int_{T}^{t} R(t-s) f\left(s, y_{s}\right) \mathrm{d} s, \quad t \in[T, 2 T] .
$$

Using Banach fixed point theorem again, we can obtain as above a fixed point $y(t)$ of $\Gamma_{1}$ on $C([T, 2 T], X)$. Hence, the function

$$
x^{2}(t)= \begin{cases}y(t) & t \in[T, 2 T] \\ x^{1}(t) & t \in(-\infty, T],\end{cases}
$$

is a mild solution of Eq. 1.1 on $(-\infty, 2 T]$. Thus, by induction, we get the solution

$$
x^{n+1}(t)= \begin{cases}x(t) & t \in[n T,(n+1) T], \\ x^{n}(t) & t \in(-\infty, n T]\end{cases}
$$

of Eq. [1.1) on $(-\infty,(n+1) T]$ for all $n \geq 1$. Therefore, we infer that there is a mild solution of Eq. (1.1) on $(-\infty, \infty)$ for any $\phi \in \mathscr{B}$.

Now, we verify that the uniqueness of mild solutions for Eq. (1.1) by using Gronwall's lemma. Let $u(t, \phi)$ and $v(t, \phi)$ be two solutions of Eq. (1.1) through $(0, \phi)$, then, for each $t \in[0, \infty)$,

$$
\begin{aligned}
\|u(t, \phi)-v(t, \phi)\| & \leq \int_{0}^{t}\|R(t-s)\|\left\|f\left(s, u_{s}\right)-f\left(s, v_{s}\right)\right\| \mathrm{d} s \\
& \leq K^{*} L_{1} M \int_{0}^{t} \mathrm{e}^{-\gamma(t-s)} \sup _{0 \leq \tau \leq s}\|u(\tau)-v(\tau)\| \mathrm{d} s
\end{aligned}
$$

which implies that

$$
\sup _{0 \leq s \leq t}\|u(s)-v(s)\| \leq K^{*} L_{1} M \int_{0}^{t} \mathrm{e}^{-\gamma(t-s)} \sup _{0 \leq \tau \leq s}\|u(\tau)-v(\tau)\| \mathrm{d} s .
$$

Applying Gronwall's lemma, we infer $u(t)=v(t)$ for $t$ in $[0, \infty)$. Therefore, $u(t, \phi)=v(t, \phi)$ for $t \in(-\infty, \infty)$. 


\subsection{Regularity of solutions}

We devote in this subsection to the regularity of the global mild solutions for Eq. 1.1. obtained above, that is, we will provide sufficient conditions to allow the differentiability of the global mild solutions of (1.1). Let us give the definition of classical solutions of Eq. (1.1) as below.

Definition 4. A function $x(\cdot): \mathbb{R} \rightarrow D(A)$ is said to be a classical solution of Eq. [1.1), if

(1) $x \in C([0, \infty), D(A)) \cap C^{1}((0, \infty), X)$;

(2) $x$ satisfies that $x_{0}=\phi$ and

$$
\frac{\mathrm{d}}{\mathrm{d} t}\left[x(t)+\int_{0}^{t} N(t-s) x(s) \mathrm{d} s\right]=A\left[x(t)+\int_{0}^{t} F(t-s) x(s) \mathrm{d} s\right]+f\left(t, x_{t}\right) \quad \text { for } t \in[0, \infty) .
$$

The main result of this subsection is that:

THEOREM 3.2. Let $\mathscr{B}$ be a fading memory space satisfying axiom $\left(C_{1}\right)$ and suppose that the condition $\left(H_{1}\right)$ and (2.4) are fulfilled. Assume further that the function $f \in C^{1}([0, \infty) \times \mathscr{B}, X)$, and the partial derivatives $D_{1} f(\cdot, \cdot), D_{2} f(\cdot, \cdot)$ are both Lipschitz continuous with respect to the second variables, i.e., there exists $l_{i}>0$ such that

$$
\left\|D_{i} f\left(t, x_{1}\right)-D_{i} f\left(t, x_{2}\right)\right\| \leq l_{i}\left\|\phi_{1}-\phi_{2}\right\|_{\mathscr{B}},
$$

for any $t \in[0, \infty), \phi_{1}, \phi_{2} \in \mathscr{B}, i=1,2$. Then, for any initial function $\phi \in \mathscr{B}$ which is continuously differentiable with $\phi(0) \in D(A)$, the mild solution $x(\cdot)$ given by Theorem 3.1 is also a classical solution of Eq. 1.1.

Pr o of. Let $T>0$ (being arbitrary), from Theorem 3.1 the unique mild solution $x(\cdot)$ of Eq. 1.1 through $(0, \phi)$ is given by

$$
x(t)=R(t) \phi(0)+\int_{0}^{t} R(t-s) f\left(s, x_{s}\right) \mathrm{d} s .
$$

Proceeding as in the proof of Theorem 3.1 , one can easily show that there exists a unique solution $y(\cdot) \in C([0, T], X)$ to the following linear integral equation on $(-\infty, T]$,

$$
y(t)=\left\{\begin{array}{l}
R(t)[A \phi(0)+f(0, \phi)]-\frac{\mathrm{d}}{\mathrm{d} t} \int_{0}^{t} R(t-s) N(s) \phi(0) \mathrm{d} s+\int_{0}^{t} R(t-s) A F(s) \phi(0) \mathrm{d} s \\
\quad+\int_{0}^{t} R(t-s)\left[D_{1} f\left(s, x_{s}\right)+D_{2} f\left(s, x_{s}\right) y_{s}\right] \mathrm{d} s, \quad t \in[0, T], \\
\phi^{\prime}(t), \quad t \in(-\infty, 0] .
\end{array}\right.
$$

We now introduce the function $z(t)$ as

$$
z(t)= \begin{cases}\phi(0)+\int_{0}^{t} y(s) \mathrm{d} s, & t \in[0, T], \\ \phi(t), & t \in(-\infty, 0] .\end{cases}
$$

By Lemma 2.2, we get that

$$
z_{t}=\phi+\int_{0}^{t} y_{s} \mathrm{~d} s, \quad t \in[0, T]
$$


and we will show $x(\cdot)=z(\cdot)$ on $[0, T]$, which implies immediately that $x(\cdot)$ is continuously differentiable on $[0, T]$ as $z(\cdot)$ is so. Indeed, it follows from $(3.5)$ and $(3.6)$ that

$$
\begin{aligned}
z(t)= & \phi(0)+\int_{0}^{t} R(s)[A \phi(0)+f(0, \phi)] \mathrm{d} s-\int_{0}^{t} R(t-s) N(s) \phi(0) \mathrm{d} s \\
& +\int_{0}^{t} \int_{0}^{s} R(s-\tau) A F(\tau) \phi(0) \mathrm{d} \tau \mathrm{d} s+\int_{0}^{t} \int_{0}^{s} R(s-\tau)\left[D_{1} f\left(\tau, x_{\tau}\right)+D_{2} f\left(\tau, x_{\tau}\right) y_{\tau}\right] \mathrm{d} \tau \mathrm{d} s .
\end{aligned}
$$

From 3.7. the function $t \rightarrow z_{t}$ and $t \rightarrow \int_{0}^{t} R(t-s) f\left(s, z_{s}\right) \mathrm{d} s$ are continuously differentiable, consequently, we see that

$$
\begin{aligned}
\frac{\mathrm{d}}{\mathrm{d} t} \int_{0}^{t} R(t-s) f\left(s, z_{s}\right) \mathrm{d} s & =\frac{\mathrm{d}}{\mathrm{d} t} \int_{0}^{t} R(s) f\left(t-s, z_{t-s}\right) \mathrm{d} s \\
& =R(t) f(0, \phi)+\int_{0}^{t} R(t-s)\left[D_{1} f\left(s, z_{s}\right)+D_{2} f\left(s, z_{s}\right) y_{s}\right] \mathrm{d} s,
\end{aligned}
$$

which implies

$$
\int_{0}^{t} R(s) f(0, \phi) \mathrm{d} s=\int_{0}^{t} R(t-s) f\left(s, z_{s}\right) \mathrm{d} s-\int_{0}^{t} \int_{0}^{s} R(s-\tau)\left[D_{1} f\left(\tau, z_{\tau}\right)+D_{2} f\left(\tau, z_{\tau}\right) y_{\tau}\right] \mathrm{d} \tau \mathrm{d} s .
$$

And, on the other hand, put $x=\phi(0)$ in 2.3 and integrating from 0 to $t$, we find

$$
\phi(0)+\int_{0}^{t} R(s) A \phi(0) \mathrm{d} s=R(t) \phi(0)+\int_{0}^{t} R(t-s) N(s) \phi(0) \mathrm{d} s-\int_{0}^{t} \int_{0}^{s} R(s-\tau) A F(\tau) \phi(0) \mathrm{d} \tau \mathrm{d} s .
$$

Substituting (3.9) and (3.10) into (3.8), we then obtain that

$$
\begin{aligned}
z(t)= & R(t) \phi(0)+\int_{0}^{t} R(t-s) f\left(s, z_{s}\right) \mathrm{d} s+\int_{0}^{t} \int_{0}^{s} R(s-\tau)\left[D_{1} f\left(\tau, x_{\tau}\right)-D_{1} f\left(\tau, z_{\tau}\right)\right] \mathrm{d} \tau \mathrm{d} s \\
& +\int_{0}^{t} \int_{0}^{s} R(s-\tau)\left[D_{2} f\left(\tau, x_{\tau}\right)-D_{2} f\left(\tau, z_{\tau}\right)\right] y_{\tau} \mathrm{d} \tau \mathrm{d} s .
\end{aligned}
$$

Hence, it follows from (3.4) that

$$
\begin{aligned}
z(t)-x(t)= & \int_{0}^{t} R(t-s)\left(f\left(s, z_{s}\right)-f\left(s, x_{s}\right)\right) \mathrm{d} s \\
& +\int_{0}^{t} \int_{0}^{s} R(s-\tau)\left[D_{1} f\left(\tau, x_{\tau}\right)-D_{1} f\left(\tau, z_{\tau}\right)\right] \mathrm{d} \tau \mathrm{d} s \\
& +\int_{0}^{t} \int_{0}^{s} R(s-\tau)\left[D_{2} f\left(\tau, x_{\tau}\right)-D_{2} f\left(\tau, z_{\tau}\right)\right] y_{\tau} \mathrm{d} \tau \mathrm{d} s .
\end{aligned}
$$


So, from the conditions $\left(H_{1}\right)$ and $(3.3)$ it yields that

$$
\begin{aligned}
\|x(t)-z(t)\| \leq & \int_{0}^{t}\|R(t-s)\|\left\|f\left(s, z_{s}\right)-f\left(s, x_{s}\right)\right\| \mathrm{d} s \\
& +\int_{0}^{t} \int_{0}^{s}\|R(s-\tau)\|\left\|D_{1} f\left(\tau, x_{\tau}\right)-D_{1} f\left(\tau, z_{\tau}\right)\right\| \mathrm{d} \tau \mathrm{d} s \\
& +\int_{0}^{t} \int_{0}^{s}\|R(s-\tau)\|\left\|D_{2} f\left(\tau, x_{\tau}\right)-D_{2} f\left(\tau, z_{\tau}\right)\right\|\left\|y_{\tau}\right\| \|_{\mathscr{B}} \mathrm{d} \tau \mathrm{d} s \\
\leq & \left(K^{*} L_{1} M+K^{*} l_{1} M T+\left(K^{*}\|y\|_{C}+M^{*}\left\|\phi^{\prime}\right\|_{\mathscr{B}}\right) K^{*} l_{2} M T\right) \int_{0}^{t} \sup _{0 \leq \tau \leq s}\|x(\tau)-z(\tau)\| \mathrm{d} s
\end{aligned}
$$

or

$$
\begin{aligned}
\sup _{0 \leq s \leq t}\|x(s)-z(s)\| \leq & \left(K^{*} L_{1} M+K^{*} l_{1} M T+\left(K^{*}\|y\|_{C}+M^{*}\left\|\phi^{\prime}\right\|_{\mathscr{B}}\right) K^{*} l_{2} M T\right) \\
& \times \int_{0}^{t} \sup _{0 \leq \tau \leq s}\|x(\tau)-z(\tau)\| \mathrm{d} s .
\end{aligned}
$$

Then, applying Gronwall lemma, we conclude that $x(t)=z(t)$ for all $t \in[0, T]$ and $x(t)$ is continuously differentiable on $[0, T]$, and hence the following formula holds

$$
x^{\prime}(t)=R^{\prime}(t) \phi(0)+f\left(t, x_{t}\right)+\int_{0}^{t} R^{\prime}(t-s) f\left(s, x_{s}\right) \mathrm{d} s .
$$

In order to prove $x(\cdot)$ is a classical solution of Eq. 1.1 on $[0, T]$, we only need to testify that it satisfies Eq. (1.1). Using (3.4), we see that

$$
\begin{aligned}
& \frac{\mathrm{d}}{\mathrm{d} t}\left[x(t)+\int_{0}^{t} N(t-s) x(s) \mathrm{d} s\right] \\
& =\frac{\mathrm{d}}{\mathrm{d} t}\left[R(t) \phi(0)+\int_{0}^{t} R(t-s) f\left(s, x_{s}\right) \mathrm{d} s+\int_{0}^{t} N(t-s)\left[R(s) \phi(0)+\int_{0}^{s} R(s-\tau) f\left(\tau, x_{\tau}\right) \mathrm{d} \tau\right] \mathrm{d} s\right] \\
& =\frac{\mathrm{d}}{\mathrm{d} t}\left[R(t) \phi(0)+\int_{0}^{t} N(t-s) R(s) \phi(0) \mathrm{d} s\right] \\
& \quad+\frac{\mathrm{d}}{\mathrm{d} t}\left[\int_{0}^{t} R(t-s) f\left(s, x_{s}\right) \mathrm{d} s+\int_{0}^{t} N(t-s) \int_{0}^{s} R(s-\tau) f\left(\tau, x_{\tau}\right) \mathrm{d} \tau \mathrm{d} s\right] \\
& :=I_{1}+I_{2} .
\end{aligned}
$$

From 2.2 it follows that

$$
I_{1}=A\left[R(t) \phi(0)+\int_{0}^{t} F(t-s) R(s) \phi(0) \mathrm{d} s\right] .
$$


As for $I_{2}$, we write

$$
I_{2}=\frac{\mathrm{d}}{\mathrm{d} t}\left[\int_{0}^{t} R(t-s) f\left(s, x_{s}\right) \mathrm{d} s\right]+\frac{\mathrm{d}}{\mathrm{d} t}\left[\int_{0}^{t} N(t-s) \int_{0}^{s} R(s-\tau) f\left(\tau, x_{\tau}\right) \mathrm{d} \tau \mathrm{d} s\right]:=I_{21}+I_{22} .
$$

According to 3.11, we have

$$
I_{21}=f\left(t, x_{t}\right)+\int_{0}^{t} R^{\prime}(t-s) f\left(s, x_{s}\right) \mathrm{d} s
$$

and, by 2.2 again,

$$
\begin{aligned}
I_{22}= & \frac{\mathrm{d}}{\mathrm{d} t}\left(\int_{0}^{t} \int_{\tau}^{t} N(t-s) R(s-\tau) f\left(\tau, x_{\tau}\right) \mathrm{d} s \mathrm{~d} \tau\right) \\
= & \frac{\mathrm{d}}{\mathrm{d} t}\left(\int_{0}^{t} \int_{0}^{t-\tau} N(t-\tau-s) R(s) f\left(\tau, x_{\tau}\right) \mathrm{d} s \mathrm{~d} \tau\right) \\
= & A \int_{0}^{t} R(t-s) f\left(s, x_{s}\right) \mathrm{d} s-\int_{0}^{t} R^{\prime}(t-s) f\left(s, x_{s}\right) \mathrm{d} s \\
& +A \int_{0}^{t} F(t-s) \int_{0}^{s} R(s-\tau) f\left(\tau, x_{\tau}\right) \mathrm{d} \tau \mathrm{d} s .
\end{aligned}
$$

We substitute (3.14) and 3.15) into 3.13) yield that

$$
I_{2}=A \int_{0}^{t} R(t-s) f\left(s, x_{s}\right) \mathrm{d} s+A \int_{0}^{t} F(t-s) \int_{0}^{s} R(s-\tau) f\left(\tau, x_{\tau}\right) \mathrm{d} \tau \mathrm{d} s+f\left(t, x_{t}\right) .
$$

Combining (3.12) and (3.16) we then obtain

$$
\begin{aligned}
\frac{\mathrm{d}}{\mathrm{d} t}\left[x(t)+\int_{0}^{t} N(t-s) x(s) \mathrm{d} s\right]= & A R(t) \phi(0)+A \int_{0}^{t} F(t-s) R(s) \phi(0) \mathrm{d} s+A \int_{0}^{t} R(t-s) f\left(s, x_{s}\right) \mathrm{d} s \\
& +A \int_{0}^{t} F(t-s) \int_{0}^{s} R(s-\tau) f\left(\tau, x_{\tau}\right) \mathrm{d} \tau \mathrm{d} s+f\left(t, x_{t}\right) \\
= & A\left(R(t) \phi(0)+\int_{0}^{t} R(t-s) f\left(s, x_{s}\right) \mathrm{d} s\right) \\
& +A \int_{0}^{t} F(t-s)\left(R(s) \phi(0)+\int_{0}^{s} R(s-\tau) f\left(\tau, x_{\tau}\right) \mathrm{d} \tau\right) \mathrm{d} s+f\left(t, x_{t}\right) \\
= & A\left[x(t)+\int_{0}^{t} F(t-s) x(s) \mathrm{d} s\right]+f\left(t, x_{t}\right) .
\end{aligned}
$$




\section{EXISTENCE AND ASYMPTOTIC PERIODICITY OF SOLUTIONS}

Finally, as $T$ is arbitrary, we conclude that $x(\cdot)$ is a classical solution of Eq. 11.1$)$ on $[0,+\infty)$, which is the desired result. The proof is completed now.

\section{Asymptotic periodicity of solutions}

The goal of this section is to establish the existence and uniqueness of $S$-asymptotically $\omega$ periodic mild solutions for the equation (1.1). Initially we introduce some concepts and properties related to $S$-asymptotically $\omega$-periodic functions.

Definition 5. A function $f \in C_{b}([0, \infty), X)$ is called to be $S$-asymptotically $\omega$-periodic if there exists $\omega>0$ such that

$$
\lim _{t \rightarrow \infty}\|f(t+\omega)-f(t)\|=0
$$

We denote by $S A P_{\omega}(X)$ the set of all $S$-asymptotically $\omega$-periodic functions from $[0, \infty)$ to $X$. Note that $S A P_{\omega}(X)$ is a Banach space with the sup-norm $\|\cdot\|_{\infty}$.

Definition 6. A continuous function $f:[0, \infty) \times X \rightarrow X$ is said to be uniformly $S$-asymptotically $\omega$-periodic on bounded sets if for each bounded subset $\mathrm{K}$ of $\mathrm{X}$, the set $\{f(t, x):(t, x) \in[0, \infty) \times K\}$ is bounded, and $\lim _{t \rightarrow \infty}(f(t+\omega, x)-f(t, x))=0$ uniformly in $x \in K$.

Definition 7. A continuous function $f:[0, \infty) \times X \rightarrow X$ is said to be asymptotically uniformly continuous on bounded sets if for every $\varepsilon>0$ and any bounded set $K \subseteq X$, there exist constants $L_{\varepsilon, K} \geq 0$ and $\delta=\delta_{\varepsilon, K}>0$ such that $\|f(t, x)-f(t, y)\| \leq \varepsilon$ for all $t \geq L_{\varepsilon, K}$ and $x, y \in K$ with $\|x-y\| \leq \delta_{\varepsilon, K}$.

The following two results, established respectively in 25] and 26], will play an essential role in proving the $S$-asymptotically $\omega$-periodicity of solutions for Eq. (1.1).

Lemma 4.1 (cf. 25: Lemma 4.1]). Let $X, Y$ be two Banach spaces, and $f:[0, \infty) \times X \rightarrow Y$ be a function uniformly $S$-asymptotically $\omega$-periodic on bounded sets and asymptotically uniformly continuous on bounded sets. If $x \in S A P_{\omega}(X)$. Then

$$
\lim _{t \rightarrow \infty}(f(t+\omega, x(t+\omega))-f(t, x(t))=0 .
$$

Lemma 4.2 (cf. [26. Lemma 2.10]). Assume that $\mathscr{B}$ is a fading memory space. Let $x: \mathbb{R} \rightarrow X$ be a function with $x_{0} \in \mathscr{B}$ and $x_{\left.\right|_{[0, \infty)}} \in S A P_{\omega}(X)$. Then the function $t \rightarrow x_{t}$ belongs to $S A P_{\omega}(\mathscr{B})$.

Next we give the definition of $S$-asymptotically $\omega$-periodic mild solutions for Eq. 1.1.

Definition 8. A bounded function $x: \mathbb{R} \rightarrow X$ is said to be $S$-asymptotically $\omega$-periodic mild solution of Eq. 11.1], if $x_{0}=\phi, x_{\left.\right|_{[0, \infty)}} \in S A P_{\omega}(X)$ and it verifies the integral equation

$$
x(t)=R(t) \phi(0)+\int_{0}^{t} R(t-s) f\left(s, x_{s}\right) \mathrm{d} s \quad \text { for } t \geq 0 .
$$

Now, we establish the first result of this part. 
THEOREM 4.1. Let $\mathscr{B}$ be a fading memory space and $f \in C([0, \infty) \times \mathscr{B}, X)$ be uniformly $S$-asymptotically $\omega$-periodic and asymptotically uniformly continuous on bounded sets. Assume that $R(t)$ is a compact operator for each $t>0$, and the conditions (2.4) and (3.1) are satisfied. Also, there exists a positive function $W \in L^{1}([0, \infty))$ such that

$$
\sup _{\|\psi\|_{\mathscr{B}} \leq \rho}\|f(t, \psi)\| \leq W(\rho)
$$

for all $t \geq 0$ and $\psi \in \mathscr{B}$. Then, for any initial function $\phi \in \mathscr{B}, E q$. 1.1. has an $S$-asymptotically $\omega$-periodic mild solution provided that

$$
\varlimsup_{\rho \rightarrow \infty}\left(\rho-\frac{M W\left(\rho_{1}\right)}{\gamma}\right)=\infty
$$

where $\rho_{1}:=K^{*} \rho+M^{*}\|\phi\|_{\mathscr{B}}$.

Proof. Let $S A P_{\omega}^{0}(X)=\left\{x \in S A P_{\omega}(X): x(0)=\phi(0)\right\}$ and the operator $\Gamma: S A P_{\omega}^{0}(X) \rightarrow$ $C([0, \infty), X)$ be defined by

$$
(\Gamma x)(t)=R(t) \phi(0)+\int_{0}^{t} R(t-s) f\left(s, x_{s}\right) \mathrm{d} s .
$$

Here we identify $x$ with its extension to $(-\infty, 0]$, by $x(\theta)=\phi(\theta)$ for $\theta \leq 0$. By virtue of (2.4) and $(3.1)$, we find that, for $x \in S A P_{\omega}^{0}(X), f\left(\cdot, x_{(\cdot)}\right)$ is bounded and hence $\Gamma x \in C_{b}([0, \infty), X)$. Moreover, from Lemma 4.1 and 4.2 , we have that $f\left(\cdot, x_{(\cdot)}\right) \in S A P_{\omega}(X)$. So, for any $\varepsilon>0$, there exists a constant $L_{\varepsilon}>0$ such that $\left\|f\left(t+\omega, x_{t+\omega}\right)-f\left(t, x_{t}\right)\right\| \leq \varepsilon$ for all $t \geq L_{\varepsilon}$.

We show at first that $\Gamma x \in S A P_{\omega}^{0}(X)$. Utilizing the conditions 2.4 and (3.1) again, we obtain that

$$
\begin{aligned}
\|(\Gamma x)(t+\omega)-(\Gamma x)(t)\| \leq & \|R(t+\omega) \phi(0)\|+\|R(t) \phi(0)\|+\int_{0}^{\omega}\|R(t+\omega-s)\|\left\|f\left(s, x_{s}\right)\right\| \mathrm{d} s \\
& +\int_{0}^{L_{\varepsilon}}\|R(t-s)\|\left\|\left(f\left(s+\omega, x_{s+\omega}\right)-f\left(s, x_{s}\right)\right)\right\| \mathrm{d} s \\
& +\int_{L_{\varepsilon}}^{t}\|R(t-s)\|\left\|\left(f\left(s+\omega, x_{s+\omega}\right)-f\left(s, x_{s}\right)\right)\right\| \mathrm{d} s \\
\leq & M \mathrm{e}^{-\gamma(t+\omega)} H\|\phi\|_{\mathscr{B}}+M \mathrm{e}^{-\gamma t} H\|\phi\|_{\mathscr{B}}+M\|f\|_{\infty} \int_{0}^{\omega} \mathrm{e}^{-\gamma(t+\omega-s)} \mathrm{d} s \\
& +M \int_{0}^{L_{\varepsilon}} \mathrm{e}^{-\gamma(t-s)}\left\|f\left(s+\omega, x_{s+\omega}\right)-f\left(s, x_{s}\right)\right\| \mathrm{d} s \\
& +M \int_{L_{\varepsilon}}^{t} \mathrm{e}^{-\gamma(t-s)}\left\|f\left(s+\omega, x_{s+\omega}\right)-f\left(s, x_{s}\right)\right\| \mathrm{d} s \\
\leq & 2 M H \mathrm{e}^{-\gamma t}\|\phi\|_{\mathscr{B}}+\frac{M\|f\|_{\infty}}{\gamma} \mathrm{e}^{-\gamma t}+\frac{2 M\|f\|_{\infty}}{\gamma} \mathrm{e}^{-\gamma\left(t-L_{\varepsilon}\right)}+\frac{M}{\gamma} \varepsilon,
\end{aligned}
$$

where $\|f\|_{\infty}=\sup _{t \geq 0}\left\|f\left(t, x_{t}\right)\right\|<\infty$. This implies that $\Gamma x \in S A P_{\omega}^{0}(X)$. 
We denote $B_{\rho}\left(0, S A P_{\omega}^{0}(X)\right)=\left\{x(\cdot) \in S A P_{\omega}^{0}(X):\|x\|_{\infty} \leq \rho\right\}$. It is clear that $B_{\rho}\left(0, S A P_{\omega}^{0}(X)\right)$ is a bounded closed convex subset of $S A P_{\omega}^{0}(X)$. Next we prove that the operator $\Gamma$ has a fix point on $B_{\rho_{0}}\left(0, S A P_{\omega}^{0}(X)\right)$ for some $\rho_{0}>0$ (determined below) by applying Schauder fixed point theorem. To do so, we certify $\Gamma$ is a completely continuous operator on $B_{\rho_{0}}\left(0, S A P_{\omega}^{0}(X)\right)$.

We claim that there exists $\rho_{0}>0$ such that $\Gamma\left(B_{\rho_{0}}\left(0, S A P_{\omega}^{0}(X)\right)\right) \subset B_{\rho_{0}}\left(0, S A P_{\omega}^{0}(X)\right)$. Indeed, if it is not true, then for each $\rho>0$, there exists a function $x_{\rho}(\cdot) \in B_{\rho}\left(0, S A P_{\omega}^{0}(X)\right)$, but $\Gamma x_{\rho}(\cdot) \notin$ $B_{\rho}\left(0, S A P_{\omega}^{0}(X)\right)$, that is $\left\|\left(\Gamma x_{\rho}\right)(t)\right\|>\rho$ for some $t:=t(\rho) \in[0, \infty)$ (dependent on $\rho$ ). On the other hand, however, in view of (4.1) and $\left\|x_{t}\right\|_{\mathscr{B}} \leq \rho_{1}$, we find that

$$
\begin{aligned}
\rho<\left\|\left(\Gamma x_{\rho}\right)(t)\right\| & \leq M H\|\phi\|_{\mathscr{B}}+M \int_{0}^{t} \mathrm{e}^{-\gamma(t-s)} W\left(\rho_{1}\right) \mathrm{d} s \\
& \leq M H\|\phi\|_{\mathscr{B}}+\frac{M W\left(\rho_{1}\right)}{\gamma}
\end{aligned}
$$

or

$$
\rho-\frac{M W\left(\rho_{1}\right)}{\gamma}<M H\|\phi\|_{\mathscr{B}}
$$

which contradicts 4.2 . Hence there is a $\rho_{0}>0$ such that $\Gamma\left(B_{\rho_{0}}\left(0, S A P_{\omega}^{0}(X)\right)\right) \subset B_{\rho_{0}}\left(0, S A P_{\omega}^{0}(X)\right)$.

To show the complete continuity of the operator $\Gamma$, we first note that it is clearly continuous on $B_{\rho_{0}}\left(0, S A P_{\omega}^{0}(X)\right)$ by the dominated convergence theorem.

Then we show that $\Gamma\left(B_{\rho_{0}}\left(0, S A P_{\omega}^{0}(X)\right)\right)=\left\{\Gamma x: x \in B_{\rho_{0}}\left(0, S A P_{\omega}^{0}(X)\right)\right\}$ is equicontinuous in $S A P_{\omega}^{0}(X)$. To do so, let $t_{1}, t_{2} \in(0, \infty)$ and $\varepsilon>0$ with $0<\varepsilon<t_{1}<t_{2}<\infty$, then for any $x \in B_{\rho_{0}}\left(0, S A P_{\omega}^{0}(X)\right)$,

$$
\begin{aligned}
\left\|(\Gamma x)\left(t_{1}\right)-(\Gamma x)\left(t_{2}\right)\right\| \leq & \left\|\left[R\left(t_{2}\right)-R\left(t_{1}\right)\right] \varphi(0)\right\|+\left\|\int_{0}^{t_{1}-\varepsilon}\left[R\left(t_{2}-s\right)-R\left(t_{1}-s\right)\right] f\left(s, x_{s}\right) \mathrm{d} s\right\| \\
& +\left\|\int_{t_{1}-\varepsilon}^{t_{1}}\left[R\left(t_{2}-s\right)-R\left(t_{1}-s\right)\right] f\left(s, x_{s}\right) \mathrm{d} s\right\|+\left\|\int_{t_{1}}^{t_{2}} R\left(t_{2}-s\right) f\left(s, x_{s}\right) \mathrm{d} s\right\| \\
\leq & \left\|\left[R\left(t_{2}\right)-R\left(t_{1}\right)\right] \varphi(0)\right\|+\int_{0}^{t_{1}-\varepsilon}\left\|R\left(t_{2}-s\right)-R\left(t_{1}-s\right)\right\| W\left(\rho_{1}\right) \mathrm{d} s \\
& +2 M W\left(\rho_{1}\right) \varepsilon+M W\left(\rho_{1}\right)\left|t_{2}-t_{1}\right| .
\end{aligned}
$$

As $t_{2} \rightarrow t_{1}$ and $\varepsilon$ sufficiently small, the right-hand side of the above inequality tends to zero independently of $x \in B_{\rho_{0}}\left(0, S A P_{\omega}^{0}(X)\right)$, since $R(t)$ is strong continuous and the compactness of $R(t)$ for $t>0$ implies the continuity in the uniform operator topology on $(0, \infty)$. Thus, $\Gamma$ maps $B_{\rho_{0}}\left(0, S A P_{\omega}^{0}(X)\right)$ into an equicontinuous family of functions on $[0, \infty)$ (note $(\Gamma x)(0)=\phi(0)$ ).

Now, we prove that $\left(\Gamma B_{\rho_{0}}\left(0, S A P_{\omega}^{0}(X)\right)\right)(t)=\left\{(\Gamma x)(t): x \in B_{\rho_{0}}\left(0, S A P_{\omega}^{0}(X)\right)\right\}$ is relatively compact in $X$ for each $t \in[0, \infty)$. Obviously, $\left(\Gamma B_{\rho_{0}}\left(0, S A P_{\omega}^{0}(X)\right)\right)(0)$ is relatively compact in $X$. Let $t \in(0, \infty)$ be fixed, $0<\varepsilon<t$, for $x \in B_{\rho_{0}}\left(0, S A P_{\omega}^{0}(X)\right)$, we define

$$
\left(\Gamma_{\varepsilon} x\right)(t)=R(t) \phi(0)+\int_{0}^{t-\varepsilon} R(t-s) f\left(s, x_{s}\right) \mathrm{d} s .
$$


Since $R(t)(t>0)$ is compact, we infer that $\left(\Gamma_{\varepsilon} B_{\rho_{0}}\left(0, S A P_{\omega}^{0}(X)\right)\right)(t)$ is relatively compact in $X$. Furthermore, for $x \in B_{\rho_{0}}\left(0, S A P_{\omega}^{0}(X)\right)$, we have

$$
\left\|(\Gamma x)(t)-\left(\Gamma_{\varepsilon} x\right)(t)\right\| \leq \int_{t-\varepsilon}^{t}\left\|R(t-s) f\left(s, x_{s}\right)\right\| \mathrm{d} s \leq M W\left(\rho_{1}\right) \varepsilon,
$$

which implies that $\left(\Gamma B_{\rho_{0}}\left(0, S A P_{\omega}^{0}(X)\right)\right)(t)$ is totally bounded, i.e, relatively compact in $X$. From the Arzelà-Ascoli theorem, we deduce that $\Gamma$ is a completely continuous map. Hence, from Schauder's fixed point theorem there exists a fixed point $x(\cdot)$ on $S A P_{\omega}^{0}(X)$ for the operator $\Gamma$. Now, set

$$
x^{*}(t)= \begin{cases}x(t), & t \in[0, \infty), \\ \phi(t), & t \in(-\infty, 0],\end{cases}
$$

then $x^{*}(t)$ is clearly a $S$-asymptotically $\omega$-periodic mild solution of problem 1.1 on $(-\infty, \infty)$. The proof is finished.

If $(R(t))_{t \geq 0}$ doesn't verify the compactness condition, we can prove the existence and uniqueness of $S$ asymptotically $\omega$-periodic solutions by Banach fixed point theorem under lipschitz conditions. That is,

$\left(H_{2}\right)$ The function $f:[0, \infty) \times \mathscr{B} \rightarrow X$ is continuous and there exist a measurable bounded function $L_{2} \in L^{1}\left([0, \infty), \mathbb{R}^{+}\right)$such that

$$
\left\|f\left(t, \phi_{1}\right)-f\left(t, \phi_{2}\right)\right\| \leq L_{2}(t)\left\|\phi_{1}-\phi_{2}\right\|_{\mathscr{B}}, \quad t \in[0, \infty), \phi_{1}, \phi_{2} \in \mathscr{B} .
$$

THEOREM 4.2. Let $\mathscr{B}$ be a fading memory space and $f$ be uniformly $S$-asymptotically $\omega$-periodic on bounded sets. Suppose that (2.4) and the assumption $\left(H_{2}\right)$ hold. Then, for any $\phi \in \mathscr{B}$, the Cauchy problem (1.1) has a unique $S$-asymptotically $\omega$-periodic mild solution on $(-\infty, \infty)$.

Proof. Let $S A P_{\omega}^{0}(X)=\left\{x \in S A P_{\omega}(X): x(0)=\phi(0)\right\}$ and the operator $\Gamma: S A P_{\omega}^{0}(X) \rightarrow$ $C_{b}([0, \infty), X)$ given by (4.3). By (2.4) and the condition $\left(H_{2}\right)$, we have that $f(\cdot, \cdot)$ is a bounded function. Furthermore, $\|\Gamma x\|_{\infty} \leq M H\|\phi\|_{\mathscr{B}}+\frac{M\|f\|_{\infty}}{\gamma}$, where $\|f\|_{\infty}=\sup _{t \geq 0}\left\|f\left(t, x_{t}\right)\right\|$. In addition, from Lemma 4.1 and 4.2 we also infer that $f\left(\cdot, x_{(\cdot)}\right) \in S A P_{\omega}(X)$. Hence, for arbitrary $\varepsilon>0$, there exists constant $L_{\varepsilon}>0$ such that $\left\|f\left(t+\omega, x_{t+\omega}\right)-f\left(t, x_{t}\right)\right\| \leq \varepsilon$ for all $t \geq L_{\varepsilon}$.

As in the proofs of the previous theorem, we can readily prove that $\Gamma x \in S A P_{\omega}^{0}(X)$. In the sequel we show that $\Gamma^{n}$ is a contractive map. Let $x, y \in S A P_{\omega}^{0}(X)$, due to the condition $\left(H_{2}\right)$ and (2.4) we first have that

$$
\begin{aligned}
\|(\Gamma x)(t)-(\Gamma y)(t)\| & \leq M \int_{0}^{t} L_{2}(s)\left\|x_{s}-y_{s}\right\|_{\mathscr{B}} \mathrm{d} s \\
& \leq K^{*} M\left\|L_{2}\right\|_{L^{1}}\|x-y\|_{\infty},
\end{aligned}
$$

for all $0 \leq t<\infty$. Put $u=\Gamma x$ and $v=\Gamma y$, from (4.4) we further get that

$$
\begin{aligned}
\left\|\left(\Gamma^{2} x\right)(t)-\left(\Gamma^{2} y\right)(t)\right\| & =\|(\Gamma u)(t)-(\Gamma v)(t)\| \\
& \leq K^{*} M \int_{0}^{t} L_{2}(s) \sup _{0 \leq \tau \leq s}\|u(\tau)-v(\tau)\| \mathrm{d} s \\
& \leq \frac{\left(K^{*} M\left\|L_{2}\right\|_{L^{1}}\right)^{2}}{2 !}\|x-y\|_{\infty},
\end{aligned}
$$




\section{EXISTENCE AND ASYMPTOTIC PERIODICITY OF SOLUTIONS}

for each $t \in[0, \infty)$. Proceeding inductively, we then obtain that

$$
\left\|\left(\Gamma^{n} x\right)(t)-\left(\Gamma^{n} y\right)(t)\right\| \leq \frac{\left(K^{*} M\left\|L_{2}\right\|_{L^{1}}\right)^{n}}{n !}\|\| x-y \|_{\infty} .
$$

Noting that $\frac{\left(K^{*} M\left\|L_{2}\right\|_{L^{1}}\right)^{n}}{n !}<1$ for $n$ large enough, we deduce $\Gamma^{n}$ is contractive and hence by Banach fixed point principle $\Gamma$ has a fixed point in $S A P_{\omega}^{0}(X)$. Let

$$
x^{*}(t)= \begin{cases}x(t) & t \in[0, \infty) \\ \phi(t) & t \in(-\infty, 0]\end{cases}
$$

then $x^{*}(t)$ is a $S$-asymptotically $\omega$-periodic solution of problem 1.1 on $(-\infty, \infty)$.

Finally, the uniqueness of $S$-asymptotically $\omega$-periodic mild solutions for Eq. (1.1) can be readily verified by using Gronwall lemma. The proof is completed.

\section{An example}

In this section, we apply the obtained abstract results to investigate the existence and asymptotical periodicity of solutions for the following neutral partial functional integro-differential equation which arises in the theory of viscoelastic materials (see [8, 10,20,31]).

$$
\left\{\begin{array}{c}
\frac{\partial}{\partial t}\left[z(t, x)-\int_{0}^{t}(t-s) \mathrm{e}^{-(t-s)} z(s, x) \mathrm{d} s\right]= \\
\quad+a(t) \int_{-\infty}^{t} \int_{0}^{\pi} h(s-t, x, z(s, y)) \mathrm{d} y \mathrm{~d} s, \quad 0 \leq x \leq \pi, t \geq 0 \\
z(t, 0)=z(t, \pi)=0, \quad t \in[0, \infty), \\
z(\theta, x)=\phi(\theta, x), \quad \theta \in(-\infty, 0], x \in[0, \pi]
\end{array}\right.
$$

where the functions $a(\cdot), f_{1}(\cdot, \cdot)$ and $\phi(\cdot, \cdot)$ will be described below.

We first need to rewrite this system into the form of Eq. 1.1). For this, let the space $X=$ $L^{2}([0, \pi], \mathbb{R})$ and the operator $(A, D(A))$ be defined by

$$
A z=z^{\prime \prime}
$$

with the domain

$$
D(A)=\left\{z(\cdot) \in X: z^{\prime}, z^{\prime \prime} \in X \text { and } z(0)=z(\pi)=0\right\} .
$$

Then $A$ generates a strongly continuous semigroup $(T(t))_{t>0}$ which is compact, analytic, and self-adjoint. Moreover, $A$ has a discrete spectrum, the eigenvalues are $-n^{2}, n \in \mathbb{N}$, with the corresponding normalized eigenvectors $\xi_{n}(x)=\sqrt{\frac{2}{\pi}} \sin (n x), n=1,2, \ldots$ And, for $z \in D(A)$,

$$
A z=\sum_{n=1}^{\infty}-n^{2}\left\langle z, \xi_{n}\right\rangle \xi_{n}
$$

Here we choose the phase space as $\mathscr{B}=\mathcal{C}_{g}^{0}$, where the space $\mathcal{C}_{g}^{0}$ is defined in the following way. Let $g:(-\infty, 0] \rightarrow(0, \infty)$ be a continuous function satisfying $g(\theta) \rightarrow \infty$ as $\theta \rightarrow-\infty$, and the function $G(t)=\sup _{\theta \leq-t} \frac{g(t+\theta)}{g(\theta)}$ is locally bounded for $t \geq 0$, then

$$
\mathcal{C}_{g}^{0}=\left\{\phi \in C((-\infty, 0], X): \lim _{\theta \rightarrow-\infty} \frac{\|\phi(\theta)\|}{g(\theta)}=0\right\},
$$


and its norm is given by

$$
\|\phi\|_{g}=\sup _{\theta \leq 0} \frac{\|\phi(\theta)\|}{g(\theta)}
$$

for $\phi \in \mathcal{C}_{g}^{0}$. According to 23, it is known that $C_{g}^{0}$ satisfies the axioms $(A),\left(A_{1}\right),(B),\left(C_{1}\right)$ and $\left(C_{2}\right)$, and in this situation we can take $H=1, K(t)=1$ and $M(t)=G(t)$. Moreover, if $G(\cdot)$ is a bounded function, then $C_{g}^{0}$ is a fading memory space.

We assume that the following conditions hold:

$\left(a_{1}\right)$ The function $h:(-\infty, 0] \times[0, \pi] \times \mathbb{R} \rightarrow \mathbb{R}$ is continuously differentiable and there exists a measurable function $b_{1}(\cdot, \cdot):(-\infty, 0] \times[0, \pi] \rightarrow \mathbb{R}^{+}$such that

$$
\left|h\left(\theta, x, y_{1}\right)-h\left(\theta, x, y_{2}\right)\right| \leq b_{1}(\theta, x)\left|y_{1}-y_{2}\right|
$$

and

$$
|h(\theta, x, y)| \leq b_{1}(\theta, x)|y|
$$

for $\theta \in(-\infty, 0], x \in[0, \pi]$ and $y_{1}, y_{2}, y \in \mathbb{R}$. Moreover, there holds

$$
L:=\int_{0}^{\pi}\left(\int_{-\infty}^{0} g(\theta) b_{1}(\theta, x) \mathrm{d} \theta\right)^{2} \mathrm{~d} x<\infty .
$$

$\left(a_{2}\right)$ The initial function $\phi$ defined by $\phi(\theta)(x)=\phi(\theta, x)$ belongs to $C_{g}^{0}$.

We now take $u(t)(x)=z(t, x)$, and define the functions $N(t): X \rightarrow X, F(t): D(A) \subseteq X \rightarrow$ $D(A)$ and $f:[0, \infty) \times C_{g}^{0} \rightarrow X$, respectively, as

$$
\begin{aligned}
(F(t) u)(x) & =\mathrm{e}^{-t} u(x), \\
(N(t) u)(x) & =-t \mathrm{e}^{-t} u(x),
\end{aligned}
$$

for $u(\cdot) \in X$ and

$$
f(t, \phi)(x)=a(t) \int_{-\infty}^{0} \int_{0}^{\pi} h(\theta, x, \phi(\theta)(y)) \mathrm{d} y \mathrm{~d} \theta \quad \text { for } \phi \in C_{g}^{0} .
$$

Then, with these notations, system (5.1) are rewritten into the form of (1.1). From [37], it is easy to see that the conditions $\left(V_{2}\right)-\left(V_{4}\right)$ are all verified with $\hat{N}(\lambda)=-\frac{1}{(\lambda+1)^{2}} I, \hat{F}(\lambda)=\frac{1}{\lambda+1} I$ in this situation. As a result, the corresponding linear system of (5.1) has an operator $(R(t))_{t \geq 0}$, which is given by

$$
R(t) z=\left\{\begin{array}{l}
\frac{1}{2 \pi \mathrm{i}} \int_{\Gamma_{r, \vartheta}} \mathrm{e}^{\lambda t}(\lambda I+\lambda \hat{N}(\lambda)-A-A \hat{F}(\lambda))^{-1} z \mathrm{~d} \lambda, \quad t>0, z \in X \\
I, \quad t=0
\end{array}\right.
$$

where $\Gamma_{r, \vartheta}$ is as described in Section 2. Moreover, by virtue of the property of $(\lambda I-A)^{-1}$, it is seen that $R(t)$ is compact for all $t>0$ since the semigroup $(T(t))_{t \geq 0}$ is so. Additionally, employing the similar arguments as in $\left[37\right.$, we can certify that $W_{G}=-\frac{1}{2}$ and hence there exist constants $M \geq 1$ and $\gamma>0$ such that $\| \overrightarrow{R(} t) \| \leq M \mathrm{e}^{-\gamma t}$ for all $t \geq 0$.

In the sequel we assume that $G(\cdot)$ is a bounded function so that $C_{g}^{0}$ is a fading memory space. We have that, 


\section{EXISTENCE AND ASYMPTOTIC PERIODICITY OF SOLUTIONS}

(1) If the function $a(\cdot) \in C^{1}\left(\mathbb{R}^{+}, \mathbb{R}\right)$ and it is $S$-asymptotically $\omega$-periodic, then the assumption $\left(a_{1}\right)$ implies clearly that the function $f$ satisfies the conditions of Theorem 3.1 and Theorem 3.2. Particularly, from 5.2 and direct computations it is easy to find that the Lipschitz constant $L_{1}$ in $\left(H_{1}\right)$ for this case given by $L_{1}=\|a\|_{\infty} \sqrt{\pi L}$ since $a(\cdot)$ is continuous and bounded due to its $S$-asymptotic periodicity. Therefore, by virtue of Theorems 3.1 and 3.2 , for any initial function $\phi$ which is continuously differentiable on $[0,+\infty)$ with $\phi(0, x) \in D(A)$, the system (5.1) admits a unique classical solution on $(-\infty,+\infty)$. Furthermore, from Theorem 4.1 the solutions are meanwhile $S$-asymptotically periodic provided that $(4.2)$ is satisfied (here $W(\rho)=L_{1} \rho$ ).

(2) Let now $a(\cdot)$ be $S$-asymptotically $\omega$-periodic on $[0,+\infty)$ and belongs to $L^{1}\left([0, \infty), \mathbb{R}^{+}\right)$. Then $\left(H_{2}\right)$ holds true with $L_{2}(t)=a(t) \sqrt{\pi L}$. As a consequence, Eq. 5.1 admits a unique $S$ asymptotically $\omega$-periodic solution for any $\phi \in C_{g}^{0}$.

Acknowledgement. We would like to thank the referees greatly for the careful review and the valuable comments on this paper.

\section{REFERENCES}

[1] AGARWAL, R. P.-CUEVAS, C.-SOTO, H.-EL-GEBEILY, M.: Asymptotic periodicity for some evolution equations in Banach spaces, Nonlinear Anal. 74 (2011), 1769-1798.

[2] AGARWAL, R. P.-DOMOSHNITSKY, A.-GOLTSER, YA.: Stability of partial functional integrodifferential equations, J. Dyn. Control Syst. 12 (2006), 1-31.

[3] CANNARSA, P.-SFORZA, D.: Global solutions of abstract semilinear parabolic equations with memory terms, NoDEA Nonlinear Differential Equations Appl. 10 (2003), 399-430.

[4] CHANG, Y.-PONCE, R.: Uniform exponential stability and applications to bounded solutions of integrodifferential equations in Banach spaces, J. Integral Equations Appl. 30 (2018), 347-369.

[5] CHEN, P.-ZHANG, X.-LI, Y.: A blowup alternative result for fractional nonautonomous evolution equation of Volterra type, Commun. Pure Appl. Anal. 17 (2018), 1975-1992.

[6] CLÉMENT, PH.-NOHEL, J. A.: Asymptotic behavior of solutions of nonlinear Volterra equations with completely positive kernels, SIAM J. Math. Anal. 12 (1981), 514-535.

[7] CLÉMENT, PH.-PRÜSS, J.: Global existence for a semilinear parabolic Volterra equation, Math. Z. 209 (1992), 17-26.

[8] COLEMAN, B. D.-GURTIN, M. E.: Equipresence and constitutive equations for rigid heat conductors, Z. Angew. Math. Phys. 18 (1967), 199-208.

[9] CUEVAS, C.-LIZAMA, C.: S-asymptotically w-periodic solutions for semilinear Volterra equations, Math. Methods Appl. Sci. 33 (2010), 1628-1636.

[10] DESCH, W.-GRIMMER, R.-SCHAPPACHER, W.: Wellposedness and wave propagation for a class of integrodifferential equations in Banach space, J. Differential Equations 74 (1988), 391-411.

[11] DIOP, M. A.-CARABALLO, T.: Existence and asymptotic behavior of solutions for neutral stochastic partial integrodifferential equations with infinite delays, Stoch. Dyn. 16 (2016), 1-17.

[12] DIMBOUR, W.-N'GUÉRÉKATA, G. M.: S-asymptotically $\omega$-periodic solutions to some classes of partial evolution equations, Appl. Math. Comp. 218 (2012), 7622-7628.

[13] ENGEL, K.-J.-NAGEL, R.: One-Parameter Semigroups for Linear Evolution Equations, Springer, New York, 2000.

[14] EZZINBI, K.-GHNIMI, S.: Existence and regularity of solutions for neutral partial functional integrodifferential equations, Nonlinear Anal. (RWA) 11 (2010), 2335-2344.

[15] EZZINBI, K.-GHNIMI, S.: Existence and regularity of solutions for some partial integrodifferential equations involving the nonlocal conditions, Numer. Funct. Anal. Optim. 40 (2019), 1532-1549.

[16] FU, X.-GAO, Y.-ZHANG, Y.: Existence of solutions for neutral integrodifferential equations with nonlocal conditions, Taiwanese J. Math. 16 (2012), 1879-1909.

[17] GRIMMER, R.: Resolvent operator for integral equations in a Banach space, Trans. Amer. Math. Soc. 273 (1983), 333-349.

[18] GRIMMER, R.-KAPPEL, F.: Series expansions of Volterra integrodifferential equations in Banach space, SIAM J. Math. Anal. 15 (1984), 595-604. 
[19] GRIMMER, R.-PRITCHARD, A. J.: Analytic resolvent operators for integral equations in a Banach space, J. Differential Equations 50 (1983), 234-259.

[20] GURTIN, M. E.-PIPKIN, A. C.: A general theory of heat conduction with finite wave speeds, Arch. Ration. Mech. Anal. 31 (1968), 113-126.

[21] HALE, J.-KATO, J.: Phase space for retarded equations with infinite delay, Funkcial. Ekvac. 21 (1978), $11-41$.

[22] HALE, J. K.-VERDUYN LUNEL, S. M.: Introduction to Functional Differential Equations, Springer-Verlag, New York, 1993.

[23] HINO, Y.-MURAKAMI, S.-NAITO, T.: Functional Differential Equations with Infinite Delay, Springerverlag, Berlin, 1991.

[24] HERNÁNDEZ, E.-PIERRI, M.: S-asymptotically $\omega$-periodic solutions for abstract equations with statedependent delay, Bull. Aust. Math. Soc. 98 (2018), 456-464.

[25] HENRÍQUEZ, H. R.-PIERRI, M.-TÁBOAS, P.: On S-asymptotically $\omega$-periodic functions on Banach spaces and applications, J. Math. Anal. Appl. 343 (2008), 1119-1130.

[26] HENRÍQUEZ, H. R.-PIERRI, M.-TÁBOAS, P.: Existence of S-asymptotically w-periodic solutions for abstract neutral equations, Bull. Aust. Math. Soc. 78 (2008), 365-382.

[27] HU, Z.- -JIN, Z.: Necessary and sufficient conditions for the regularity and stability of solutions for some partial neutral functional differential equations with infinite delay, Nonlinear Anal. 73 (2010), 2752-2765.

[28] LEUGering, G.: A Generation Result for a Class of Linear Thermo-Viscoelastic Material. In: Dynamical Problems in Mathematical Physics, Verlag Peter Lang, Frankfurt, 1983.

[29] LI, F.-WANG, H.: S-asymptotically $\omega$-periodic mild solutions of neutral fractional differential equations with finite delay in Banach space, Mediterr. J. Math. 14 (2017), 1-16.

[30] LUNARDI, A.: On the linear heat equation with fading memory, SIAM J. Math. Anal. 21 (1990), 1213-1224.

[31] MILLER, R. K.: An integrodifferential equation for rigid heat conductions with memory, J. Math. Anal. Appl. 66 (1978), 313-332.

[32] MOKKEDEM, F. Z.-FU, X.: Approximate controllability of semi-linear neutral integro-differential systems with finite delay, Appl. Math. Comp. 242 (2014), 202-215.

[33] NAITO, T.-SHIN, J. S.-MURAKAMI, S.: The generator of the solution semigroup for the general linear functional differential equation, Bull. Univ. Electro-Comm. 11 (1998), 29-38.

[34] PAZY, A.: Semigroups of Linear Operators and Applications to Partial Differential Equations, Springer-Verlag, New York, 1983.

[35] PIERRI, M.-O'REGAN, D.: S-asymptotically $\omega$-periodic solutions for abstract neutral differential equations, Electr. J. Diff. Equ. 2015 (2015), 1-14.

[36] DOS SANTOS, J. P. C.-HENRÍQUEZ, H.-HERNÁNDEZ, E.: Existence results for neutral integrodifferential equations with unbounded delay, J. Int. Equ. Appl. 23 (2011), 289-330.

[37] DOS SANTOS, J. P. C.-HENRÍQUEZ, H. R.: Existence of S-asymptotically $\omega$-periodic solutions to abstract integro-differential equations, Appl. Math. Comp. 256 (2015), 109-118.

[38] WU, J.: Theory and Applications of Partial Functional Differential Equations, Springer-Verlag, New York, 1996.

[39] XIA, Z.-WANG, D.-WEN, C.-YAO, J.: Psuedo asymptotically periodic mild solutions of semilinear functional integro-differential equations in Banach spaces, Math. Methods Appl. Sci. 40 (2017), 7333-7355.

[40] YAN, Z.-YAN, X.: The optimal behavior of solutions to fractional impulsive stochastic integro-differential equations and its control problems, J. Fixed Point Theory Appl. 21 (2019), 1-42.

Received 5. 12. 2019

Accepted 24. 6. 2021

\author{
* College of Mathematics Science \\ Inner Mongolia Normal University \\ Hohhot 010022 \\ P. R. CHINA \\ E-mail: zhujianbo789@163.com \\ ** School of Mathematical Sciences \\ Shanghai Key Laboratory of PMMP \\ East China Normal University \\ Shanghai 200241 \\ P. R. CHINA \\ E-mail:xlfu@math.ecnu.edu.cn
}

